\title{
METODOLOGIA ANALÍTICA PARA QUANTIFICAR O TEOR DE BIODIESEL NA MISTURA BIODIESEL:DIESEL UTILIZANDO ESPECTROSCOPIA NA REGIÃO DO INFRAVERMELHO
}

\author{
Lílian Lefol Nani Guarieiro* \\ Departamento de Química Analítica, Instituto de Química, Universidade Federal da Bahia, 40170-115 Salvador - BA, Brasil \\ Angelo C. Pinto \\ Departamento de Química Orgânica, Instituto de Química, Universidade Federal do Rio de Janeiro, 21945-970 \\ Rio de Janeiro - RJ, Brasil \\ Paula Fernandes de Aguiar \\ Departamento de Química Analítica, Instituto de Química, Universidade Federal do Rio de Janeiro, 21945-970 \\ Rio de Janeiro - RJ, Brasil \\ Núbia Moura Ribeiro \\ Centro Federal de Educação Tecnológica de Bahia, 40301-015 Salvador - BA, Brasil
}

Recebido em 26/2/07; aceito em 14/6/07; publicado na web em 14/1/08

\begin{abstract}
DETERMINATION OF BIODIESEL PERCENTAGE IN BIODIESEL:DIESEL MIXTURES USING MID-INFRARED SPECTROSCOPY. Considered the best substitute for diesel, biodiesel can be blended with diesel in any ratio, bringing lots of environmental, economic and social advantages. Brazilian law $\mathrm{N}^{\circ} 11097 / 2005$, proposes the introduction of biodiesel in to the Brazilian energy matrix, mixed with diesel at a minimum percentage of $2 \%$. For consumers and sellers to be sure that the commercialized mixture of biodiesel:diesel contains the correct percentage, it is necessary to develop analytical methodologies to quantify the amount of biodiesel added. This work presents a fast, low-cost and simple methodology to determine the biodiesel proportion in mixtures of biodiesel:diesel, based on infrared spectroscopy.
\end{abstract}

Keywords: biodiesel; biodiesel:diesel mixtures; mid-infrared spectroscopy.

\section{INTRODUÇÃO}

A possibilidade de uso de óleos vegetais como combustível foi reconhecida desde os primórdios dos motores diesel. O próprio Rudolph Diesel utilizou vários óleos vegetais em seus motores e no prefácio escrito por ele para o livro Termodinâmica, de Chalkey (1911), afirmou: "O motor diesel pode ser alimentado com óleos vegetais e poderá ajudar consideravelmente o desenvolvimento da agricultura nos países onde ele funcionar. Isso parece um sonho do futuro, mas eu posso predizer com inteira convicção que esse modo de emprego do motor diesel pode, em dado tempo, adquirir uma grande importância"'.

Os derivados de óleos vegetais são substitutos adequados para o óleo diesel por não requererem modificações nos motores e apresentarem alto rendimento energético. Eles não contêm enxofre (portanto, não contribuem para geração de ácido sulfuroso na atmosfera - chuva ácida) e sua combustão gera menores teores de gases poluentes que o óleo diesel, pois retiram $\mathrm{CO}_{2}$ da atmosfera para crescimento da planta e a quantidade de $\mathrm{CO}_{2}$ liberada na combustão desses óleos é menor que a gasta na sua produção. Por isso, o uso de biodiesel de origem vegetal reduz o percentual de $\mathrm{CO}_{2}$, um gás de efeito estufa, na atmosfera ${ }^{2}$.

A alternativa mais viável de transformação dos óleos vegetais para gerar um combustível capaz de fazer funcionar um motor por compressão sem danificá-lo tem sido o biodiesel. Considerado um combustível biodegradável e ambientalmente correto, o biodiesel quimicamente é uma mistura de ésteres mono-alquílicos de ácidos graxos $^{3}$. O processo mais empregado para sua produção é a trans-

*e-mail: lilianlefol@yahoo.com.br esterificação, que consiste na reação de um triglicerídeo com um álcool de cadeia curta, na presença de um catalisador ácido ou básico. Como resultado, obtém-se ésteres de ácidos graxos metílicos ou etílicos (biodiesel) e glicerina ${ }^{4}$.

Um combustível alternativo precisa ser tecnicamente competitivo, facilmente acessível e compatível com as necessidades de proteção ambiental. Em termos econômicos a produção de biodiesel, especificamente para o Brasil, possibilitará a redução nas importações de petróleo e óleo diesel refinado. Segundo estatísticas da ANP (Agência Nacional de Petróleo, Gás Natural e Biocombustíveis) o consumo brasileiro de óleo diesel apresentou um aumento acumulado de 42,5\% no período de 1992 a 2001. Para suprir a demanda crescente, foi necessário aumentar o volume da importação de 2,3 milhões de $\mathrm{m}^{3}$ em 1992 para 6,6 milhões de $\mathrm{m}^{3}$ em 2001. O Brasil importa atualmente cerca de $11 \%$ de óleo diesel. O consumo brasileiro de óleo diesel, em 2003, foi de $38.099 .000 \mathrm{~m}^{3}$, sendo 97,15\% consumido no transporte rodoviário. De acordo com a ANP, 5\% de biodiesel misturado ao óleo diesel consumido no País, representará uma economia de 380 milhões dólares/ano.

Recentemente, o governo brasileiro lançou o Programa Nacional de Produção e Uso do Biodiesel que entrou em vigor em 2005, através da Lei no 11.097 de 13 de janeiro de 2005, que incrementa a participação dos biocombustíveis na matriz energética brasileira, sendo fixado o uso de $2 \%$ de biodiesel misturado ao diesel. A adição de biodiesel nessa proporção trará uma economia de divisas da ordem de U\$ 152 milhões e a geração de 153.000 novos empregos.

A ANP estabeleceu normas de certificação da qualidade do biodiesel, e a partir de janeiro de 2008 o biodiesel especificado obrigatoriamente será distribuído na percentagem de $2 \%$ de biodiesel misturado ao diesel (B2). Para isso, há necessidade do de- 
senvolvimento de metodologias analíticas para quantificar o percentual de biodiesel nas misturas diesel:biodiesel.

A literatura científica apresenta relatos sobre o uso de espectroscopia no infravermelho (IV) para o monitoramento da transesterificação de óleos vegetais com metanol ${ }^{5,6}$ e etanol ${ }^{7}$, determinando a taxa de conversão desta reação. Como o diesel e o biodiesel apresentam funções químicas distintas, os espectros de infravermelho desses combustíveis contêm bandas específicas. Esta técnica pode ser usada também para quantificar o percentual de biodiesel presente em misturas biodiesel:diesel. De acordo com $K_{\text {nnothe }}^{8}$, a espectroscopia de infravermelho próximo associada à Ressonância Magnética Nuclear de Hidrogênio (RMN ${ }^{1} \mathrm{H}$ ), utilizando calibração multivariada, pode ser utilizada para determinar o percentual de biodiesel em mistura com diesel. Oliveira el al. ${ }^{9} \mathrm{e}$ Pimentel el al. ${ }^{10}$ utilizaram IV para determinar o teor de biodiesel misturado ao diesel através das técnicas de PLS (Mínimos Quadrados Parciais) com ANN (Rede Neural Artificial) e PCA (Análise de Componentes Principais), respectivamente.

Este trabalho apresenta o desenvolvimento de uma metodologia de análise por infravermelho, utilizando calibração univariada, para quantificar o percentual de biodiesel misturado ao diesel. É importante ressaltar que se partiu do pressuposto de que o biodiesel deverá estar dentro das especificações brasileiras (estabelecidas pela portaria 255/ 2003 da ANP), antes de ser adicionado ao diesel em distribuidoras credenciadas. Para isso, neste trabalho também foi produzido biodiesel de óleo de soja com aproximadamente $100 \%$ de pureza.

\section{PARTE EXPERIMENTAL}

\section{Produção do biodiesel}

Os ensaios para produção dos ésteres metílicos foram realizados utilizando óleo de soja comercial refinado. A transesterificação do óleo foi realizada com metanol anidro, sob as condições experimentais descritas a seguir.

Em um balão de fundo redondo acoplado a um condensador de refluxo, contendo $45,20 \mathrm{~g}$ de óleo, foram adicionados $0,26 \mathrm{mg}$ de carbonato de potássio $(3 \% \mathrm{~mol})$ e $63 \mathrm{~mL}$ de metanol. A mistura reacional foi mantida sob refluxo e agitação vigorosa, por um período de 1:30 h. Após este período, o aquecimento foi desligado e assim que a mistura reacional alcançou a temperatura ambiente, o excesso de metanol foi removido por evaporação à pressão reduzida.

Os produtos foram separados em funil de separação. A fase contendo o glicerol foi separada e desprezada, carreando consigo o excesso do álcool e o catalisador. A fase contendo o produto desejado foi lavada primeiramente com hexano para evitar a formação de emulsão. Em seguida, a fase hexânica foi lavada com água para remover qualquer catalisador residual e outros contaminantes. Os traços de água na fase hexânica foram removidos com sulfato de magnésio anidro. O produto foi filtrado em sílica-gel, para adsorver substâncias polares que porventura ainda existissem. O excesso do solvente foi retirado por evaporação sob pressão reduzida. Obtevese, então, biodiesel límpido, de cor amarelo claro.

A reação com óleo de soja apresentou uma excelente conversão dos triglicerídeos em ésteres metílicos (aproximadamente $100 \%$ ). A conversão foi avaliada por espectro de Ressonância Magnética Nuclear de Hidrogênio $\left(\mathrm{RMN}{ }^{1} \mathrm{H}\right)^{11}$. O biodiesel assim produzido foi considerado padrão para o método proposto.

\section{Preparação das amostras padrão}

Levando em conta a proposta do governo brasileiro de utilizar $2 \%$ de biodiesel misturado ao óleo diesel, foram preparadas amos- tras na faixa de 0,5 a $5 \%$ de biodiesel adicionado ao diesel. $\mathrm{Na}$ preparação das misturas B0,5; B1; B1,5; B2; B2,5; B3; B3,5; B4; B4,5 e B5, foram utilizados pipeta automática de $5-50 \mu \mathrm{L}$ e balões volumétricos de 2 e $4 \mathrm{~mL}$.

Para avaliar a seletividade do método proposto foram preparadas misturas biodiesel:diesel nas proporções de 25, 50 e $75 \%$ de biodiesel adicionado no diesel (estas misturas foram denominadas B25, B50 e B75). Tais misturas foram preparadas utilizando balões volumétricos de $100 \mathrm{~mL}$. O biodiesel e o diesel puro foram denominados de $\mathrm{B} 100$ e $\mathrm{B} 0$, respectivamente.

\section{Metodologia de análise por IV}

Para a determinação das concentrações das misturas biodiesel:diesel foi utilizado um espectrofotômetro de infra-vermelho (IV) da marca Nicolet, modelo Avatar 330 FT-IR, com reflectância atenuada (ATR) e porta-amostra de cristal de germânio da marca Pike de $80 \mathrm{~mm}$ de comprimento, $10 \mathrm{~mm}$ de largura e $4 \mathrm{~mm}$ de espessura. A região selecionada para análise foi infravermelho médio (MID-IR).

As amostras das misturas biodiesel:diesel foram analisadas colocando-se na cubeta do porta-amostra $0,2 \mathrm{~mL}$ de cada mistura. Foram obtidos os espectros de IV das misturas, do biodiesel puro e espectro do óleo diesel utilizado. As análises das misturas foram realizadas em triplicata, exceto para o diesel puro que foram feitas 10 replicatas.

\section{Cálculo da área da banda da carbonila de ésteres na região de ésteres $\left(\sim 1745 \mathrm{~cm}^{-1}\right)$}

A área da banda da carbonila de ésteres foi calculada com os espectros no modo Absorbância. Dessa forma, foi selecionada a região de $1850-1650 \mathrm{~cm}^{-1}$ e calculou-se a área de cada espectro, utilizando o aplicativo do equipamento OMNIC versão 6.1a, da Thermo Nicolet, que acompanha o equipamento.

\section{Parâmetros analisados para a avaliação do desempenho da metodologia}

O processo de validação de um método verifica, por meio de estudos laboratoriais, se suas características de desempenho estão de acordo com as especificações apresentadas para a intenção do uso dos resultados analíticos. Assim, de acordo com o manual de orientações sobre validação de métodos de ensaios químico do INMETRO, as características de desempenho determinadas foram: teste de seletividade, avaliação do limite de detecção (LD) e quantificação (LQ), escolha da faixa de trabalho e da faixa linear do método, estudo da sensibilidade e da linearidade do método, exatidão e precisão.

\section{RESULTADOS E DISCUSSÃO}

A espectroscopia na região do infravermelho médio é uma técnica adequada para a quantificação de biodiesel em mistura com diesel, pois a função carbonila dos ésteres metílicos ou etílicos do biodiesel é uma banda única, fina e absorve em região distinta do espectro de infravermelho do diesel. Por essas características, espectroscopia na região do IV médio foi a técnica escolhida para quantificação de biodiesel no diesel.

\section{Padrão utilizado na validação do método}

O biodiesel produzido foi considerado como padrão, pois apresentou aproximadamente $100 \%$ de ésteres metílicos. 


\section{Validação do método proposto}

\section{Seletividade}

Neste critério foi verificado se compostos presentes no diesel poderiam ser interferentes em uma determinada região do espectro de IV quando este estivesse misturado ao biodiesel.

Na Figura 1 podemos observar que o espectro do diesel puro possui aspecto diferente dos espectros da mistura de biodiesel. Isto é esperado, porque os compostos presentes no diesel são misturas de hidrocarbonetos, e não possuem substâncias carboniladas, as quais estão presentes no biodiesel. Assim, surgem algumas regiões como opção para ser desenvolvida a metodologia proposta para este trabalho, pois substâncias carboniladas, especificamente ésteres, apresentam duas absorções característica que têm origem nos estiramentos da ligação $\mathrm{C}=\mathrm{O}$ (são bastante intensas) e $\mathrm{C}-\mathrm{O}$ (razoavelmente intensas).

As vibrações de estiramento da ligação $\mathrm{C}=\mathrm{O}$ de ésteres ocorrem entre 1750 e $1735 \mathrm{~cm}^{-1} \mathrm{e}$ as da ligação $\mathrm{C}-\mathrm{O}$ de ésteres que são, na verdade, duas vibrações assimétricas acopladas: $\mathrm{C}-\mathrm{C}(=\mathrm{O})-\mathrm{O}$ e $\mathrm{O}-\mathrm{C}-\mathrm{C}$, ocorrem entre 1300 e $1000 \mathrm{~cm}^{-112}$. Porém, as correlações feitas com a vibração do estiramento da ligação $\mathrm{C}-\mathrm{O}$ são menos confiáveis que as feitas com o estiramento da ligação $\mathrm{C}=\mathrm{O}$, porque compostos que contenham hidrocarbonetos podem apresentar absorções na região em que ocorrem os estiramentos da ligação C-O (o que poderia causar uma alteração da sensibilidade do método proposto). Assim, foi escolhida a região de absorção de $\mathrm{C}=\mathrm{O}$ para o desenvolvimento da metodologia. Nesta região, o espectro de diesel não apresenta nenhuma absorção, como pode ser visualizado na Figura 1. Pode-se observar também que, com o aumento da concentração de biodiesel na mistura biodiesel:diesel, há um aumento proporcional do tamanho da banda de $\mathrm{C}=\mathrm{O}$.

Como o diesel não possui banda de carbonila, as bandas observadas no espectro das misturas na região entre 1750 e $1735 \mathrm{~cm}^{-1}$ devem ser provenientes do biodiesel. Desta forma, pode-se dizer que o método proposto é seletivo, uma vez que apesar da técnica de IV detectar vários sinais, existe uma banda específica que pode ser usada para quantificar o biodiesel. Além disso, as diferentes proporções da mistura biodiesel:diesel não apresentaram influencia da presença de interferentes. Entretanto, é preciso avaliar a aplicação do método na faixa de trabalho de interesse.

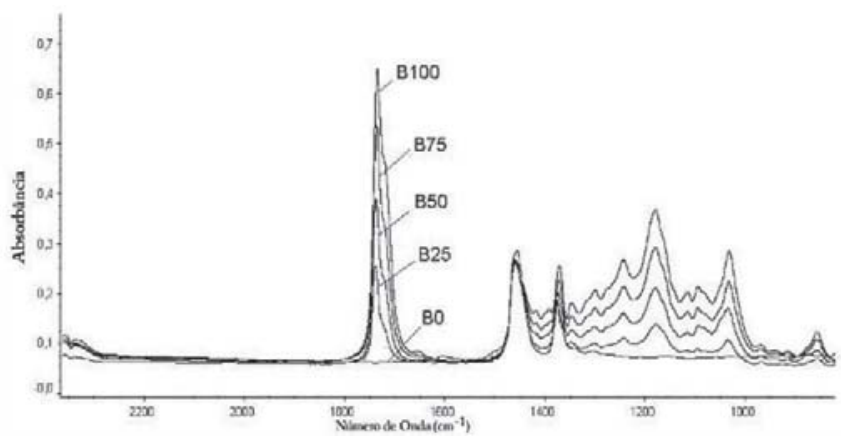

Figura 1. Espectros das misturas biodiesel:diesel BO, B25, B50, B75 e B100 na região do Infravermelho de aproximadamente 2200 a $900 \mathrm{~cm}^{-1}$

Avaliação do limite de detecção e do limite de quantificação

Os limites de detecção e quantificação foram obtidos segundo critérios estatísticos ${ }^{13-17}$. Para isso, 10 repetições do branco foram determinadas para $0,2 \mathrm{~mL}$ da matriz (diesel puro) e os limites de detecção e quantificação foram obtidos, respectivamente, segundo o critério 3 s e 10s da leitura do branco (Tabela 1). Foi avaliada a presença de possíveis valores aberrantes através do teste de Grubbs ${ }^{18}$.
Tabela 1. LD e LQ para avaliação do branco da amostra

\begin{tabular}{lcc}
\hline Leitura & Área real & Área medida \\
\hline 1 & 0 & $-0,11$ \\
2 & 0 & $-0,19$ \\
3 & 0 & $-0,19$ \\
4 & 0 & $-0,42$ \\
5 & 0 & $-0,16$ \\
6 & 0 & $-0,30$ \\
7 & 0 & $-0,09$ \\
8 & 0 & $-0,16$ \\
9 & 0 & $-0,08$ \\
10 & 0 & $-0,03$ \\
\hline & Valor real & Valor medido \\
\hline Média & 0 & $-0,185$ \\
Desvio Padrão & 0 & 0,133 \\
$\mathrm{G}_{\text {tabelado }}$ & & 2,290 \\
\hline
\end{tabular}

LD: limite de detecção; $L Q$ : limite de quantificação

Como a definição de LD é visual, considera-se que quando a área da banda é diferente de zero, é possível determiná-la. Assume-se então, que o LD é o limite de detecção do olho humano. Como qualquer alteração significativa na linha de base do espectro pode ser visualmente verificada, qualquer valor diferente de zero pode ser verificado.

Para calcular os limites de detecção e quantificação foram utilizados os valores denominados como área real e valor real. Para isso, considerou-se que não existe área negativa e assim todo valor medido negativo foi igualado a zero. Portanto, ambos os limites de detecção e de quantificação foram zero (valor da área da banda de $\mathrm{C}=\mathrm{O}$ ), o que corresponde a uma concentração da mistura biodiesel:diesel de $0,1 \%$, respectivamente, utilizando a curva de calibração da validação (Figura 2).

Através avaliação dos limites de detecção e quantificação, concluímos que a curva de calibração pode ser utilizada para quantificar o teor de biodiesel na mistura biodiesel:diesel em concentrações a partir de $0,1 \%$ de biodiesel misturado ao diesel. Isto significa que, de um modo geral, a faixa proposta (B0,5 a B5) para esta metodologia é aplicável. Levando em conta a proposta do governo brasileiro da implementação do uso da mistura B2 em 2005 e sua obrigatoriedade a partir de 2008, pode-se, através da curva de calibração determinada (Figura 2), avaliar qualquer concentração de biodiesel adicionado ao diesel acima de $0,1 \%$.

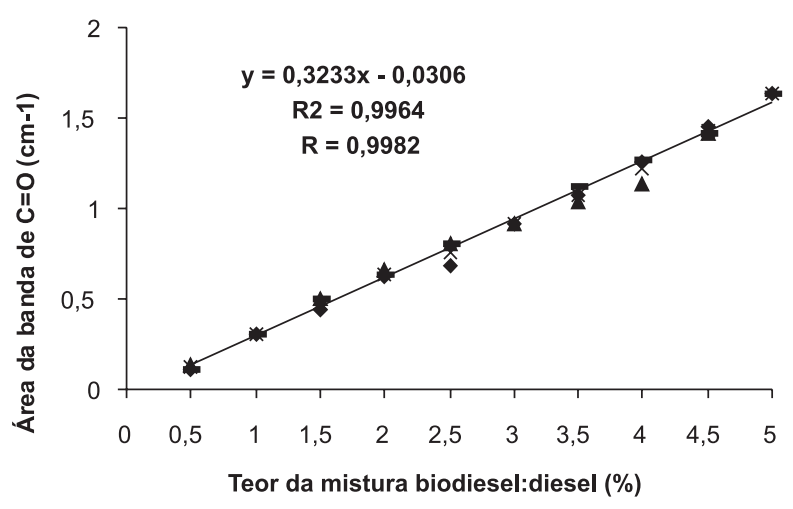

Figura 2. Relação entre as concentrações das misturas biodiesel:diesel e as áreas referente aos dados da Tabela 2 
Tabela 2. Estudo da validação das misturas biodiesel:diesel para a região de $1850-1650 \mathrm{~cm}^{-1}$

\begin{tabular}{|c|c|c|c|c|c|c|c|c|c|c|}
\hline Misturas & B 0,5 & B1 & $\mathrm{B} 1,5$ & B2 & $\mathrm{B} 2,5$ & B3 & B3,5 & B4 & $\mathrm{B} 4,5$ & B5 \\
\hline \multirow[t]{3}{*}{ Áreas } & 0,11 & 0,31 & 0,44 & 0,62 & 0,68 & 0,92 & 1,07 & 1,26 & 1,45 & 1,64 \\
\hline & 0,11 & 0,31 & 0,50 & 0,63 & 0,80 & $\theta, 94$ & 1,12 & 1,27 & 1,41 & 1,64 \\
\hline & 0,14 & $\theta, 44$ & 0,50 & 0,66 & 0,80 & 0,91 & 1,04 & 1,13 & 1,42 & \\
\hline$<\mathrm{x}_{1}>$ & 0,12 & 0,31 & 0,48 & 0,64 & 0,76 & 0,92 & 1,08 & 1,22 & 1,43 & 1,64 \\
\hline S & 0,017 & 0 & 0,036 & 0,021 & 0,069 & 0,007 & 0,041 & 0,078 & 0,021 & 0 \\
\hline$S^{2}$ & 0,001 & 0 & 0,001 & 0,001 & 0,005 & 0 & 0,002 & 0,006 & 0,001 & 0 \\
\hline DPR & 14,42 & 0 & 7,21 & 3,25 & 9,12 & 0,77 & 3,74 & 6,40 & 1,45 & 0 \\
\hline$\overline{\Sigma \mathrm{S}^{2}}$ & & & & & 0,015 & & & & & \\
\hline $\mathrm{C}_{\mathrm{cal}}$ & & & & & 0,41 & & & & & \\
\hline $\mathrm{C}_{\mathrm{tab}}^{\mathrm{cal}}$ & & & & & 0,445 & & & & & \\
\hline
\end{tabular}

Valores das misturas biodiesel:diesel correspondem às áreas da banda de $\mathrm{C}=\mathrm{O}$ em $1850-1650 \mathrm{~cm}^{-1}$. Valores de $\mathrm{x}_{1}$ : valor médio; $\mathrm{S}$ : desvio padrão; $\mathrm{S}^{2}$ variância; $\Sigma \mathrm{S}^{2}$ : somatório das variâncias; DPR: desvio padrão relativo; $\mathrm{C}_{\text {cal }}$ e $\mathrm{C}_{\text {tab }}$ : valor calculado e tabelado, respectivamente , de Cochran (95\% de confiança). Os valores tachados não atenderam ao teste de Grubbs.

Avaliação da faixa de trabalho e faixa linear do método

Determinado o limite de detecção e de quantificação, a validação parcial do método prosseguiu com a avaliação da linearidade. Este estudo baseou-se nas orientações sobre validação de métodos de ensaios químicos do Instituto Nacional de Metrologia, Normalização e Qualidade Industrial (INMETRO) ${ }^{19}$.

Todo experimento de determinação da faixa de trabalho é iniciado pela escolha de uma faixa preliminar. A escolha da faixa de trabalho foi baseada na proposta do governo brasileiro de implementação inicial da mistura B2. Selecionou-se então a faixa de 0,5 a $5 \%$, com intervalos de concentração de $0,5 \%$.

Uma sequiência de procedimentos e testes estatísticos foi aplicada aos dados experimentais obtidos, na região de 1850 - 1650 $\mathrm{cm}^{-1}$ do espectro de infravermelho, a fim de validar a curva de calibração construída (Tabela 2).

Empregou-se o ajuste dos dados ao modelo de regressão linear $(y=a+b x)$ como procedimento para construção da curva de calibração. Para isso, primeiramente, todos os dados obtidos em triplicata foram submetidos ao teste de Grubbs $(\mathrm{G})$, que se destina a verificar a existência de valores aberrantes.

A curva de calibração foi submetida ao teste de Cochan para avaliar o desvio bilateral das variâncias a um nível de significância de $5 \%$. Este teste avalia a homogeneidade das variâncias, o que permite utilizar a curva de calibração obtida com um limite de confiança aceitável. $\mathrm{O}$ valor de Cochran calculado foi menor que o valor de Cochran tabelado, mostrando variâncias homogêneas conforme o aumento da concentração. Isto caracteriza um comportamento homocedástico para esta curva de calibração.

Como a curva apresentou variâncias significativamente comparáveis, segundo o teste de Cochran, ela pode ser utilizada para fins quantitativos. Assim uma curva de calibração de concentração versus área foi construída baseando-se nos dados da Tabela 2. A reta foi ajustada ao modelo $\mathrm{y}=\mathrm{a}+\mathrm{bx}$ pelo método dos mínimos quadrados, e a análise de seu coeficiente de correlação linear (R) indicou que a dispersão dos pontos em torno da reta foi maior que 99\%, o que foi considerado razoável para esta metodologia.

Para verificar a eficiência do modelo proposto de regressão linear foi construído e analisado o gráfico de resíduos (Figura 3). O resíduo foi calculado através da diferença entre o valor observado e o valor calculado pela equação da reta, de regressão, da Figura 2, para cada valor de x. O gráfico de resíduos ilustrou uma distribuição aleatória em torno da linha reta confirmando a linearidade do método.

O gráfico de resíduos também permite observar o comportamento das variâncias em uma curva de calibração com o aumento da concentração. De acordo com a análise dos resíduos na Figura 3, pode- mos concluir que o comportamento das variâncias é homocedástico, confirmando a previsão obtida pelo teste de Cochran ${ }^{20}$.

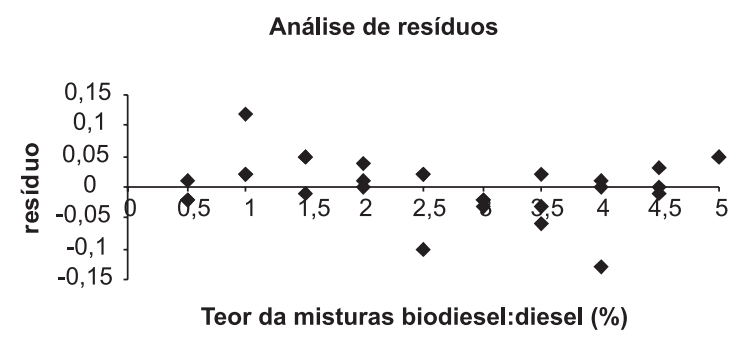

Figura 3. Gráfico de resíduos

Avaliação da exatidão do método

A avaliação da exatidão foi feita levando em consideração a avaliação da recuperação.

Avaliação da recuperação do método

A recuperação do método foi avaliada em amostras padrão preparadas com biodiesel de óleo de soja misturado ao óleo diesel nas concentrações 1,$5 ; 2$ e $2,5 \%$ de biodiesel.

Aos resultados de área obtidos foi aplicado o teste de Grubbs. De acordo com este teste, nenhum valor foi considerado aberrante e, portanto, todos os dados obtidos para uma mesma concentração puderam ser agrupados (média). A Tabela 3 mostra os valores obtidos no estudo da recuperação.

Tabela 3. Resultado do estudo da recuperação do método

\begin{tabular}{lccccc}
\hline Mistura & $\mathrm{C}_{1}$ & $\left\langle\mathrm{C}_{1}\right\rangle$ & $\mathrm{C}_{2}$ & Rend. (\%) & Er (\%) \\
\hline B1,5 & 0,44 & 0,48 & 0,45 & 106,7 & 6,7 \\
& 0,50 & & & & \\
B2 & 0,50 & & & & \\
& 0,62 & 0,64 & 0,62 & 103,2 & 3,2 \\
& 0,63 & & & & \\
B2,5 & 0,66 & & & & $-3,0$ \\
& 0,68 & 0,76 & 0,78 & 97 & \\
& 0,80 & & & & \\
\hline
\end{tabular}

* $\mathrm{C}_{1}$ : concentração da mistura obtida experimentalmente; $<\mathrm{C}_{1}>$ : média da concentração obtida experimentalmente; $\mathrm{C}_{2}$ : con-centração teórica da mistura; Rend.: rendimento; Er: erro relativo. 
Tabela 4. Estudo da validação das misturas biodiesel:diesel para a região de $1850-1650 \mathrm{~cm}^{-1}$, operador B

\begin{tabular}{lcccccccccc}
\hline Misturas & $\mathrm{B} 0,5$ & $\mathrm{~B} 1$ & $\mathrm{~B} 1,5$ & $\mathrm{~B} 2$ & $B 2,5$ & $\mathrm{~B} 3$ & $\mathrm{~B} 3,5$ & $\mathrm{~B} 4$ & $\mathrm{~B} 4,5$ & $\mathrm{~B} 5$ \\
\hline $\mathrm{S}$ & 0,007 & 0,007 & 0,007 & 0,014 & 0,016 & 0 & 0,010 & 0 & 0,020 & 0,010 \\
$\mathrm{~S}^{2}$ & 0 & 0 & 0 & 0,001 & 0 & 0 & 0 & 0 & 0,001 & 0 \\
$\mathrm{DPR}$ & 0,81 & 0,56 & 0,44 & 2,86 & 0,48 & 0 & 0,20 & 0 & 0,45 & 0,21 \\
$\Sigma \mathrm{S}^{2}$ & & & & & 0,001 & & & & & \\
$\mathrm{C}_{\text {cal }}$ & & & & & 0,40 & & & & & \\
$\mathrm{C}_{\text {tab }}$ & & & & & 0,445 & & & & & \\
\hline
\end{tabular}

Valores das misturas biodiesel:diesel correspondem às áreas da banda de $\mathrm{C}=\mathrm{O}$ em $1850-1650 \mathrm{~cm}^{-1}$. Valores de $\mathrm{x}_{1}$ : valor médio; S: desvio padrão; $\mathrm{S}^{2}$ variância; $\Sigma \mathrm{S}^{2}$ : somatório das variâncias; $\mathrm{DPR}$ : desvio padrão relativo; $\mathrm{C}_{\mathrm{cal}}$ e $\mathrm{C}_{\text {tab }}$ : valor calculado e tabelado, respectivamente, de Cochran (95\% de confiança).

$\mathrm{O}$ estudo de recuperação mostrou bons resultados. O erro relativo encontrado variou de 3,0 a $6,7 \%$, ficando abaixo de $10 \%$ para todas as concentrações analisadas; a recuperação variou de 97 a 106,7\%.

Através desses resultados, podemos concluir que o método de quantificação proposto apresentou uma boa recuperação (erro relativo $<10 \%$ ). Isto significa que para um valor indicado pela legislação igual a $0,02(2 \%)$, o método pode gerar um desvio de determinação inferior a $0,002(0,2 \%)$. $\mathrm{O}$ desvio padrão relativo variou de 3,25 a $9,12 \%$, com um desvio médio de $6,5 \%$, indicando uma boa precisão dos resultados.

Avaliação da precisão intermediária e repetitividade do método

Para avaliar a precisão do método quantitativo proposto neste trabalho, outro operador (operador B), em dia diferente, realizou o procedimento para construção de uma curva de calibração para as mesmas concentrações utilizadas no método proposto em um equipamento diferente (um espectofotômetro da marca Nicolet, MagnaIR 760, célula de $0,047 \mathrm{~mm} \mathrm{KCl}$ e no modo transmitância).

A curva de calibração foi obtida de forma análoga ao operador que havia construído a curva já apresentada, aqui denominado operador A. Para o estudo foram analisadas as misturas B0,5; B1; B1,5; B2; B2,5; B3; B3,5; B4; B4,5; B5 e diesel puro.

Através dos espectros obtidos para cada concentração, foi possível avaliar a repetitividade e a precisão intermediária do método proposto.

Analisando os dados das Tabelas 2 e 4 que correspondem às medidas obtidas para o operador A e o $\mathrm{B}$, respectivamente, verificou-se uma boa repetitividade, considerando os dados de cada operador isoladamente, apenas pequenas variações dos resultados.

Com relação à precisão intermediária, que expressa o efeito das variações das medidas com operadores diferentes e equipamentos diferentes, verificou-se que as variâncias das medidas são praticamente as mesmas quando um segundo operador executa as análises (diferença máxima de 0,5\%). Como esta variação, nesta aplicação, foi considerada pequena, pode-se dizer que o método possui uma boa precisão intermediária.

Ainda para verificar a precisão intermediária do método, foi feito um estudo comparativo das inclinações das retas obtidas pelos dois operadores.

Comparação das inclinações das retas das curvas de calibração

Para avaliar se as inclinações das curvas de calibração obtidas pelos operadores A e B são semelhantes, foi feita uma seqüência de cálculos estatísticos. Este estudo foi feito com o intuito de verificar a influência da troca de analista e tipo de equipamento nos resultados obtidos (precisão).

A Tabela 5 mostra as equações das retas obtidas para os dois operadores, assim como os valores de coeficiente de correlação linear e de variância residual de cada reta.
Tabela 5. Dados obtidos para validação do método para diferentes operadores

\begin{tabular}{lcccc}
\hline Operador & $\mathrm{n}$ & Equações & $\mathrm{R}$ & $\mathrm{Se}^{2}$ \\
\hline $\mathrm{A}$ & 10 & $\mathrm{Y}=0,3233 \mathrm{x}+0,0306$ & 0,9982 & 0,0283 \\
$\mathrm{~B}$ & 10 & $\mathrm{Y}=0,8957 \mathrm{x}+0,2988$ & 0,9979 & 0,0079 \\
\hline $\mathrm{F}_{\text {cal }}=3,59$ & \multicolumn{3}{c}{$\mathrm{F}_{\text {tab }}=3,18$} \\
$\mathrm{t}_{\text {cal }}=1,22$ & \multicolumn{3}{c}{$\mathrm{t}_{\text {cal }}=0,18$} \\
\hline
\end{tabular}

* n: número de pontos da reta; R: coeficiente de correlação linear da reta; $\mathrm{Se}^{2}$ : variância da reta; F: valor de Snedecor; t: valor de tStudent. $\left(\mathrm{t}_{\text {tabelado }}=1,860\right)$

Após o cálculo das variâncias residuais de cada reta, aplicou-se o teste de hipótese para verificar se as variâncias são significativamente iguais. A hipótese não foi aceita, pois $\mathrm{F}$ calculado foi maior que o F tabelado. Calculou-se então t', utilizando um t tabelado de $(n=2)$ graus de liberdade a um nível de significância de $5 \%^{14}$, o qual foi comparado com o t calculado. Como t' calculado foi maior que o t calculado conclui-se que as inclinações das duas retas são significativamente diferentes, havendo uma influência nos resultados devido à mudança do tipo de equipamento e operador.

Deste modo, a mudança do tipo de equipamento e do operador pode acarretar em erros maiores que o erro inerente à curva de calibração. Em função disto, este resultado mostra que os parâmetros tipo de equipamento e operador não são robustos e, portanto, as variações inerentes à troca de equipamento e operador influenciam os resultados da análise. Apesar de verificarmos que a troca de operador e equipamento simultaneamente afeta significativamente os resultados, verificou-se que, na faixa de interesse $(1,5-2,5)$, esta diferença é menor que $10 \%$, o que não deve comprometer os resultados das análises.

\section{CONCLUSÕES}

A metodologia foi proposta para quantificar o teor de biodiesel na mistura biodiesel:diesel. A faixa de concentração estudada pelo método foi de 0,5 a $5 \%$ de biodiesel adicionado no diesel, apresentando uma linearidade maior que $99 \%$.

O método mostrou-se viável para determinar o teor de biodiesel devido ao teor das misturas biodiesel:diesel a serem analisadas estar acima do limite de detecção do método, que foi de $0,1 \%$ de biodiesel.

Os resultados da recuperação do método mostraram erro relativo inferior a $10 \%$. Através desses resultados, podemos concluir que o método de quantificação proposto apresentou uma boa recuperação, pois para o valor recomendado pela legislação igual a 0,02 (2\%), o método pode gerar um desvio de determinação inferior a $0,002(0,2 \%)$. Assim, o erro relativo encontrado na metodologia proposta não acarretará em discordância para quantificar o teor da 
mistura biodiesel:diesel (B2) de determinada amostra.

Portanto, propõe-se o método de quantificação do teor de biodiesel na mistura biodiesel:diesel utilizando IV médio, como viável para quantificar o teor de biodiesel na mistura com concentrações superiores a $0,1 \%$ de biodiesel. Porém, torna-se necessária a construção de uma curva de calibração específica para cada tipo de equipamento.

\section{AGRADECIMENTOS}

Ao PRH-01 da ANP, ao CNPq e à FAPERJ pelo apoio financeiro, à L. B. Coelho, pela realização das análises, e ao CEFET Química (Nilópolis-RJ) pelo uso do espectrofotômetro.

\section{REFERÊNCIAS}

1. Sa Filho, H. L.; Rotenberg, B.; Albuquerque, S. F.; Mendonça, M. C. G.; Medeiros, P. R. S.; Informativo do INT, Rio de Janeiro, 1979, 22, 29.

2. Usta, N.; Öztürk, E.; Can, Ö.; Conkur, E. S.; Nas, S.; Con, A. H.; Can, A. Ç.; Topcu, M.; Energy Convers. Manage. 2005, 46, 741.

3. Meher, L. C.; Sagar, D. V.; Naink S. N.; Renew. Sustain. Energy Rev. 2006, $10,248$.

4 . Pinto, A. C.; Guarieiro, L. L. N.; Rezende, M. J. C.; Ribeiro, N. M.; Torres, E. A.; Lopes, W. A.; Pereira, P. A. P.; de Andrade, J. B.; J. Braz. Chem. Soc. 2005, 16, 1313.

5. Knothe, G.; J. Am. Oil Chem. Soc. 1999, 76, 795.
6. Knothe, G.; J. Am. Oil Chem. Soc. 2000, 77, 489

7. Zagonel, G. F.; Peralta-Zamora, P.; Ramos, L. P.; Talanta 2004, 63, 1021.

8. Knothe, G.; J. Am. Oil Chem. Soc. 2001, 78, 1025.

9. Oliveira, J. S.; Montalvão, R.; Daher, L.; Suarez, P. A. Z.; Rubim, J. C.; Talanta 2006, 69, 1278.

10. Pimentel, M. F.; Ribeiro, G. M. G. S.; Cruz, R. S.; Stragevitch, L.; Pacheco Filho, J. G. A.; Teixeira, L. S. G.; Microchem. J. 2006, 82, 201.

11. Gelbard, G.; Bres, O.; Vargas, R. M.; Vielfaure, F.; Schuchardt, U.; J. Am. Oil Chem. Soc. 1995, 72, 1239.

12. Silverstein, R. M.; Webster, F. X.; Identificação Espectrométrica de Compostos Orgânico, 6⿳亠 ed, LTC: Rio de Janeiro, 1998.

13. IHC; Guidance for Industry, Q2B Validation of Analytical. Procedures: Methodology, November 1996.

14. Instituto Nacional de Metrologia, Normalização e Qualidade Industrial; Orientações sobre validação de métodos de ensaios químicos, DOQCGCRE-008, Revisão: outubro/2002.

15. IUPAC Recommendation, Pure Appl. Chem. 1995, 67, 1699.

16. Ribani, M.; Bottoli, C. B. G.; Collins, C. H.; Jardim, I. C. F. S.; Melo, L. F. C.; Quim. Nova 2004, 27, 771.

17. ISO 11843; Capability of detection, International Standards Organisation, Geneva.

18. Miller, J. C.; Miller, J. N.; Estatística para química analítica, $2^{\text {nd }}$ ed., Addison-Wesley Wilmington: Delaware, USA, 1993.

19. Instituto Nacional de Metrologia, Normalização e Qualidade Industrial; Orientações sobre validação de métodos de ensaios químicos, DQOCGCRE-008, Revisão 01, março 2003.

20. Massart, D. L; Vandeginste, B. G. M.; Buydens, L. M. C.; Jong, S.; Lewi, P. J.; Smeyers-Verbeke, J.; Handbook of chemometrics and qualimetrics: part A, Elsevier: New York, 1997, vol. 20A. 